safety of stress MPS with a physiologist-led protocol using Regadenoson as the primary vasodilator agent, compared with an earlier physician-led protocol.

Methods Our centre prefers dynamic stress and will combine it with vasodilator stress as required. Earlier local audit data suggested Regadenoson was safer than other pharmacological stressors in keeping with published data. This resulted in the introduction of physiologist-led stress MPS in September 2011 and replacement of the previous physician-led protocol. Data were prospectively stored on a database. We reviewed all documented serious adverse events (AE) (VF/VT, cardiac arrest, unplanned admission, death) associated with dynamic and vasodilator stress MPS between 1 September 2008 and 30 August 2014. Outcome data between the two periods were analysed: physician-led (September 2008 to August 2011) and physiologist-led (September 2011 to August 2014).

Results In all, 11297 patients underwent stress MPS (physician-led, 5321; physiologist-led, 5976). Compared to the physician-led period, there were fewer patients in sinus rhythm in the physiologist-led period $(86.4 \%$ vs $91.4 \%, \mathrm{P}<$ 0.01 ) and more patients deemed to have a high prognostic cardiovascular risk post-scan $(11.7 \%$ vs $9.2 \%, \mathrm{P}<0.01)$ (Table 1). Vasodilator stress was used more often in the physiologist-led period $(43.9 \%$ vs $27.9 \%, \mathrm{P}<0.01)$. Regadenoson was the main vasodilator in physiologist-led period (64.9\%); Dipyridamole in physician-led period (83.9\%) (Table 1). There were 8 cases of serious $\mathrm{AE}$ (4 in each period) (0.07\%): VF/ VT ( 1 and 3 cases in physician and physiologist-led periods respectively), cardiac arrest $(2,2)$, admission $(2,3)$ and one death $(1,0)$. Relative risk of serious $\mathrm{AE}$ was 0.89 (95\% CI: 0.2-3.6) for patients in the physiologist-led period, relative to those in the physician-led period (Table 2).

Conclusions This retrospective study compared physiologist-led stress MPS using regadenoson as the primary vasodilator agent with a previous physician-led protocol. Physiologist-led stress MPS was not associated with an increased rate of serious AE, with no deaths. Regadenoson stress MPS may be used safely in a physiologist-led setting without immediate physician presence.

Abstract 116 Table 1 Demographics and Stress mechanism

\begin{tabular}{llll}
\hline & Physician-led & Physiologist-led & P-Value \\
\hline Demographics & & & \\
$\mathrm{N}$ & 5321 & 5976 & \\
Male & $56.6 \%$ & $60.1 \%$ & $<0.01$ \\
Age (median) & 66 & 66 & $\mathrm{NS}$ \\
Sinus rhythm & $91.4 \%$ & $86.4 \%$ & $<0.01$ \\
Prognostic risk post-MPS high $¥$ & $9.2 \%$ & $11.7 \%$ & $<0.01$ \\
Prognostic risk post-MPS low $\%$ & $73.0 \%$ & $68.8 \%$ & $<0.01$ \\
Stress mechanism & & & $<0.01$ \\
Physical (treadmill/bicycle) & $72.1 \%$ & $56.1 \%$ & $<0.01$ \\
Pharmacological (all) & $27.9 \%$ & $43.9 \%$ & $<0.01$ \\
Dobutamine & $10.6 \%$ & $1.1 \%$ & $<0.01$ \\
Dipyridamole & $83.9 \%$ & $29.8 \%$ & $<0.01$ \\
Adenosine & $5.4 \%$ & $4.2 \%$ & $<0.01$ \\
Regadenoson & $0.2 \%$ & $64.9 \%$ & \\
\hline
\end{tabular}

$\ddagger$ prognostic risk calculated post-MPS - high: $>3 \%$ estimated annualised risk of major adverse cardiovascular event, low: $<1 \%$
Abstract 116 Table 2 Serious adverse events

\begin{tabular}{llll}
\hline & Physician-led & Physiologist-led & \\
\hline Total cases & $4(0.1 \%)$ & $4(0.1 \%)$ & $0.9(0.2-3.6) \dagger$ \\
VFNT & 1 & 3 & \\
Cardiac arrest & 2 & 2 & \\
Emergency admission & 2 & 3 & \\
Death & 1 & 0 & \\
\hline
\end{tabular}

$\dagger 95 \%$ confidence interval

\section{OPTIMISATION AND REPRODUCIBILITY OF 18F- FLUORIDE POSITRON EMISSION TOMOGRAPHY AND COMPUTED TOMOGRAPHY IN PATIENTS WITH AORTIC STENOSIS}

Tania Pawade*, Timothy Cartlidge, David Newby, Dweck Marc. University of Edinburgh; *Presenting Author

\subsection{6/heartjnl-2016-309890.117}

Background 18F-Fluoride positron emission tomography (PET) and computed tomography (CT) can measure disease activity and progression in aortic stenosis. We aimed to optimise imaging methodology, analysis and scan-rescan reproducibility.

Methods Fifteen patients with aortic stenosis underwent $18 \mathrm{~F}$ fluoride PET-CT twice within 1 month. We compared nongated PET and non-contrast CT, with a modified approach that incorporated contrast CT and ECG-gated PET. We explored a range of image analysis techniques including estimation of blood pool activity at differing vascular sites and a most-diseased segment (MDS) approach.

Results Contrast-enhanced ECG-gated PET-CT provided superior spatial localisation of $18 \mathrm{~F}$-fluoride uptake that permitted localisation to individual valve leaflets (Figure). Scan-rescan reproducibility was markedly improved using enhanced analysis techniques leading to a reduction in variability from $25 \%$ to $<10 \%$ (tissue-to-background MDS: mean value 1.55, difference 0.05 , limits of agreement $-0 \cdot 10$ to $0 \cdot 20$ ).

Conclusion Optimised 18F-fluoride PET-CT provides excellent spatial resolution and scan-rescan reproducibility. It holds major promise as a marker of disease activity in aortic stenosis and has major potential as a biomarker end-point of trials of novel therapies in aortic stenosis.

\section{SINGLE SOURCE, DUAL ENERGY CT FOR THE ASSESSMENT OF CALCIFIED CORONARY ARTERY DISEASE}

${ }^{1}$ Benjamin Clayton, ${ }^{2}$ Franchesca Wotton*, ${ }^{2}$ Carl Roobottom, ${ }^{2}$ Gareth Morgan-Hughes. ${ }^{1}$ Torbay and South Devon NHS Foundation Trust; ${ }^{2}$ Derriford Hospital; * Presenting Author

10.1136/heartjnl-2016-309890.118

Introduction Arterial calcification can limit the visualisation of vessel lumen at CT coronary angiography (CTCA). Dual energy CT (DECT) using two x-ray spectra of differing energy, either from distinct sources or using novel detectors and a single source, offers novel approaches to this problem. It allows the generation of images depicting objects as if they have been subjected to a specific photon energy $(\mathrm{keV})$ rather than a polychromatic beam (virtual monochromatic images). With improved material identification, based on its attenuation coefficient at each $\mathrm{keV}$ and transformation into a linear combination of the two basis materials (material decomposition), it 